\title{
ESTIMATION OF WATER CONTENT IN CELLULOSE MATERIALS
}

\author{
MAXIM LAZARENKO, ALEXANDER ALEKSEEV, YURIY ZABASHTA, \\ SERGEY TKACHEV, VALERIY KOVALCHUK, DMITRIY ANDRUSENKO, \\ YURIY GRABOVSKY and LEONID BULAVIN
}

\author{
Taras Shevchenko National University of Kyiv, 60, Volodymyrska Str., \\ Kyiv, UA 01033, Ukraine \\ Corresponding author: S. Tkachov, tkachevserg@gmail.com
}

Received October 29, 2019

We have developed a method that allows determining the relative water quantity in amorphous and crystalline areas of cellulose materials using X-ray and thermogravimetric experimental data. In the present paper, by using this method, we showed that the amount of water in the crystalline region may reach $50 \%$ of the water amount that amorphous regions contain. Also, we built a structural model of water molecules that can occur in a crystal lattice.

Keywords: cellulose material, X-ray method, cellulose crystallite, swelling

\section{INTRODUCTION}

Many technical issues, such as those involved in developing wastewater remediation methods, concern the interaction between cellulose and water. ${ }^{1}$ That is why, it is important to understand how water interacts with cellulose materials. It is known that a cellulose material contains alternating crystalline and amorphous phases, and thus, it is possible to consider it as a biphasic system. ${ }^{2}$ We think that it is rational to study the water-cellulose interaction mechanism in two stages. In the first stage, the water quantity that penetrates in the two types of areas is determined. In the second stage, the molecular mechanism of interaction is studied.

The ability of water molecules to make hydrogen bonds with cellulose chains lies at the basis of this interaction. The infiltration of water molecules into the space between the chains leads to a redistribution of the hydrogen bonds in the areas adjacent to these water molecules and changes the structure of these areas. ${ }^{3}$ We think that this redistribution is different for crystalline and amorphous areas because of the differences in their initial structure.

Is there a possibility for water molecules to penetrate crystalline areas? At the first glimpse, the ordered structure of these areas excludes such a scenario. However, literature data bring evidence in favor of penetration. Warwicker et al. discussed the function of sheets of cellulose chains in the swelling reactions of cellulose. ${ }^{4}$ In the study by Papkov et al., it was noted that the transition from cellulose I to cellulose II cannot happen without the intracrystalline swelling stage, when water is present in crystalline areas. ${ }^{5}$ More recent works have also reported evidence about the penetration of water molecules into cellulose crystallites or modelled cellulose-water interactions in crystalline areas.,

As mentioned above, a cellulose material may be considered as a diphasic system because it contains alternating crystalline and amorphous areas. The latter have large free volumes because of their disorder. These free volumes are partially realized as voids. The existence of voids has as consequence the fact that water molecules fill these voids in the first place, when the cellulose material is in contact with water. At the same time, there is an opinion that the order in the crystalline areas excludes water penetration into these areas. ${ }^{2,4}$ Agarwal et al. show the size of cellulose crystallites depends on the presence of water in cellulose materials of different types. ${ }^{7}$ These two different points of view are a good basis for further investigation provided in this work. In this study, we not only concentrate on the questions whether water penetrates cellulose crystallites or not, but also we try to estimate the water quantity in the crystalline and amorphous areas and to propose a method that would allow fulfilling this task using X-ray and thermogravimetric data. 


\section{Thermodynamic aspect of water location in cellulose materials}

We will start by analyzing this situation from the thermodynamic point of view. We will consider that the crystalline and amorphous areas are in different thermodynamic phases and assign numbers 1 and 2 to the thermodynamic phases of the crystalline and amorphous areas, respectively.

A cellulose chain is the result of cellobiosis units being connected to each other. We can mark the number of cellobiosis units as $N_{1}$ and $N_{2}$, the number of water molecules in the crystalline and amorphous phases as $n_{1}$ and $n_{2}$, and the concentrations of water in the respective phases as $c_{1}$ and $c_{2}$. As a result, we obtain:

$$
\begin{aligned}
& c_{1}=\frac{n_{1}}{N_{1}+n_{1}} \\
& c_{2}=\frac{n_{2}}{N_{2}+n_{2}}
\end{aligned}
$$

Water distribution between the crystalline and amorphous phases or, in other words, the relation between $c_{1}$ and $c_{2}$ concentrations is in agreement with the condition of phase equilibrium in thermodynamics. The mentioned condition is the equality of water chemical potentials in both phases: ${ }^{12}$

$\psi_{1}(p, T)+k_{B} T \ln c_{1}=\psi_{2}(p, T)+k_{B} T \ln c_{2}$

where $\psi_{1}(p, T)$ and $\psi_{2}(p, T)$ are functions of pressure $p$ and temperature $T$, while $k_{B}$ is the Boltzmann coefficient.

The cellulose-water interaction, in general, and the difference of the cellulose-water interaction in the amorphous and crystalline regions are taken into account when $\psi_{1}(p, T)$ and $\psi_{2}(p, T)$ are calculated.

Equation (3) can be rewritten as:

$c_{2} / c_{1}=\exp \left(-\frac{\psi_{2}-\psi_{1}}{k_{B} T}\right)$

Formula (4) is the evidence that, in principle, water may enter the crystalline area of a cellulose material.

In the following, we will refer to the ratio $c_{2} / c_{1}$ as the distribution index.

\section{Connection between distribution index and microscopic characteristics of cellulose materials}

Let us mark the masses of a cellulose chain unit and a water molecule as $m_{c}$ and $m_{w}$, and the volumes they take up as $v_{c}$ and $v_{w}$. Then, we obtain formula (5) for the ratio $\omega$ of the mass of the substance in the crystalline areas and the whole mass of a system:

$$
\omega=\frac{m_{c} N_{1}+m_{w} n_{1}}{m_{c}\left(N_{1}+N_{2}\right)+m_{w}\left(n_{1}+n_{2}\right)}
$$

as well as formula (6) for the ratio of the mass of water in the system and the entire mass of the system:

$$
\xi=\frac{m_{w}\left(n_{1}+n_{2}\right)}{m_{c}\left(N_{1}+N_{2}\right)+m_{w}\left(n_{1}+n_{2}\right)}
$$

Also, we have expression (7) for the relative change in the volume of crystalline areas $\theta$ that are caused by water absorption:

$\theta=\frac{v_{w} n_{1}}{v_{c} N_{1}}$

It is possible to determine the values of ratios $\omega, \xi, \theta$, using the experimental data, as will be shown later. Now, our task is to make the ratio $c_{2} / c_{1}$ the function of microscopic characteristics of the cellulose material $\omega, \xi, \theta$.

If we divide expression (2) by equality (1), we obtain:

$\frac{c_{2}}{c_{1}}=\frac{n_{2}}{n_{1}} \frac{N_{1}}{N_{2}} \frac{1+\frac{n_{1}}{N_{1}}}{1+\frac{n_{2}}{N_{2}}}$

Then, expressions (5-7) can be rewritten in the following form:

$$
\begin{aligned}
& \omega=\frac{m_{c}+m_{w} \frac{n_{1}}{N_{1}}}{m_{c}\left(1+\frac{N_{2}}{N_{1}}\right)+\frac{n_{1}}{N_{1}} m_{w}\left(1+\frac{n_{2}}{n_{1}}\right)} \\
& \xi=\frac{\frac{n_{1}}{N_{1}} m_{w}\left(1+\frac{n_{2}}{n_{1}}\right)}{m_{c}\left(1+\frac{N_{2}}{N_{1}}\right)+\frac{n_{1}}{N_{1}} m_{w}\left(1+\frac{n_{2}}{n_{1}}\right)} \\
& \frac{n_{1}}{N_{1}}=\theta \frac{v_{c}}{v_{w}}=q
\end{aligned}
$$

If we substitute equality (11) in formulas (9, $10)$ and denote

$N_{2} / N_{1}=\eta, n_{2} / n_{1}=\varphi$

we will get another form of formulas (9) and (10):

$$
\omega=\frac{m_{c}+m_{w} q}{m_{c}(1+\eta)+q m_{w}(1+\varphi)}
$$


$\xi=\frac{q m_{w}(1+\varphi)}{m_{c}(1+\eta)+q m_{w}(1+\varphi)}$

Expressions 13 and 14 form the system of equations with variables $\varphi$ and $\eta$. Its solution is:

$\varphi=\frac{\xi}{\omega}\left(\frac{m_{c}}{m_{w}} \frac{1}{q}+1\right)-1$

$\eta=\frac{1-\xi}{\omega}\left(1+\frac{m_{w}}{m_{c}} q\right)-1$

Let's rewrite the ratio $n_{2} / N_{2}$ in variables $\varphi$ and $\eta$. For this task, we will use the identity:

$\frac{n_{2}}{n_{1}}=\frac{n_{2}}{n_{1}} \frac{N_{1}}{N_{1}} \frac{N_{2}}{N_{2}}$,

which leads to equality

$\frac{n_{2}}{N_{2}}=\frac{\varphi q}{\eta}$

Taking into consideration $(8,12)$ and $(17)$, we have the formula for the ratio $c_{2} / c_{1}$ :

$\frac{c_{2}}{c_{1}}=\frac{\varphi}{\eta} \frac{1+q}{1+\frac{q \varphi}{\eta}}$

If we substitute equalities $(11,15)$ and (16) into formula (18), we will obtain:

$\frac{c_{2}}{c_{1}}=\frac{\frac{\xi}{\omega}\left(\frac{m_{c}}{m_{w}} \frac{v_{w}}{\theta v_{c}}+1\right)-1}{\frac{1-\xi}{\omega}\left(\frac{m_{w}}{m_{c}} \frac{\theta v_{c}}{v_{w}}+1\right)-1} \cdot \frac{1+\frac{\theta v_{c}}{v_{w}}}{1+\frac{\theta v_{c}}{v_{w}} \frac{\frac{\xi}{\omega} \frac{m_{c}}{\frac{m_{w}}{m_{w}}} \frac{v_{w}}{\omega}+\frac{\xi}{\omega}\left(\frac{m_{w}}{m_{c}} \frac{\theta v_{c}}{v_{w}}+1\right)-1}{v_{w}}+1}$
The chemical formula of the cellulose chain unit is $\mathrm{C}_{6} \mathrm{H}_{10} \mathrm{O}_{5}$, so $m_{c}=162 \mathrm{D}$ and accordingly $m_{c} / m_{w}=162 / 18=9$.

The crystalline lattice of cellulose I, which is usually found in cellulose materials, has the parameters $a=6.717 \mathrm{~A}, \quad b=5.962 \mathrm{~A}$, $c=10.400 \mathrm{~A}, \gamma=80.37^{\circ}$.

In Figure 1, we depict the projection of elementary cells of such a lattice on the plane $a b$ (chain axes are shown by circles). According to this picture, area $S$ of a figure $A^{\prime} B^{\prime} C^{\prime} D^{\prime}$ is the area per one chain (points $A^{\prime}, B^{\prime}, C^{\prime}$, and $D^{\prime}$ divide line segments $\mathrm{AB}, \mathrm{BC}, \mathrm{CD}$ and $\mathrm{AD}$ in two). Considering the cell size, we get $S=20 \mathrm{~A}^{2}$.

There are two units of cellulose chains in a cell, which means that each unit takes $h=5.2 \mathrm{~A}$ along a chain. For the unit volume, we have the value $v_{c}=S h=104 \mathrm{~A}^{3}$.

The water molecule volume is $30 \AA^{3}$. Accordingly, the ratio $v_{c} / v_{w}$ has a value of 3.4. Taking mentioned values into consideration, we may rewrite formula (18) as:

$$
\frac{c_{2}}{c_{1}}=\frac{\frac{\xi}{\omega}\left(\frac{9}{3.4 \theta}+1\right)-1}{\frac{1-\xi}{\omega}\left(\frac{3.4 \theta}{9}+1\right)-1} \cdot \frac{1+3.4 \theta}{1+3.4 \theta \frac{\frac{\xi}{\omega}\left(\frac{9}{3.4 \theta}+1\right)-1}{\frac{1-\xi}{\omega}\left(\frac{3.4 \theta}{9}+1\right)-1}}
$$

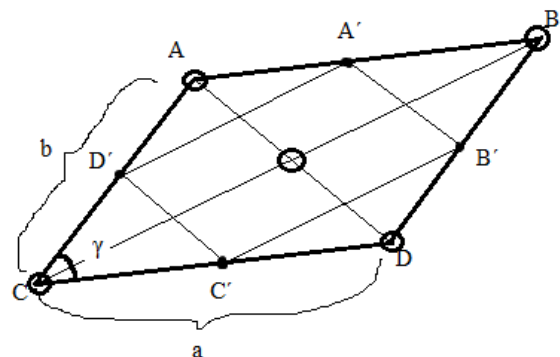

Figure 1: Projection of an elementary cell of cellulose I on the plane $a b$

\section{EXPERIMENTAL}

We explored two sample series, A and B. Sample series A was MCC of Cellets 100 brand produced by Shin Etsu Company (Japan). We ground the MCC in an agate mortar. Sample series B was filtering paper No 390 provided by Filtrak firm (Germany).

We kept the samples in a desiccator for 3 days at a temperature of $115{ }^{\circ} \mathrm{C}$ to remove moisture. Samples with different values of humidity were obtained by keeping them in an atmosphere of saturated water vapor during different time intervals. More details about the preparation of the samples can be found in our previous articles. ${ }^{9,10,11}$

The index $\xi$ was determined using the results of the thermogravimetric research. For this research, we used a Q-1500D derivatograph. ${ }^{11}$ The measurements were made in the temperature interval $\mathrm{T}=20 \div 250{ }^{\circ} \mathrm{C}$, at the heating speed of $5{ }^{\circ} \mathrm{C} / \mathrm{min}$. 
The values of $\omega$ and $\theta$ were determined using Xray diffraction analysis. The X-ray diffraction patterns were obtained on a DRON-3M X-ray diffractometer, provided with a BSV-28 tube $(\lambda=1.54178 \AA){ }^{10,11}$

\section{RESULTS AND DISCUSSION}

The obtained values of $\xi$ are presented in Table 1. $\xi$ is equal to 0.003 for dried samples. Figures 2 and 3 present the diffractograms of the samples of series A and B. They are dependences

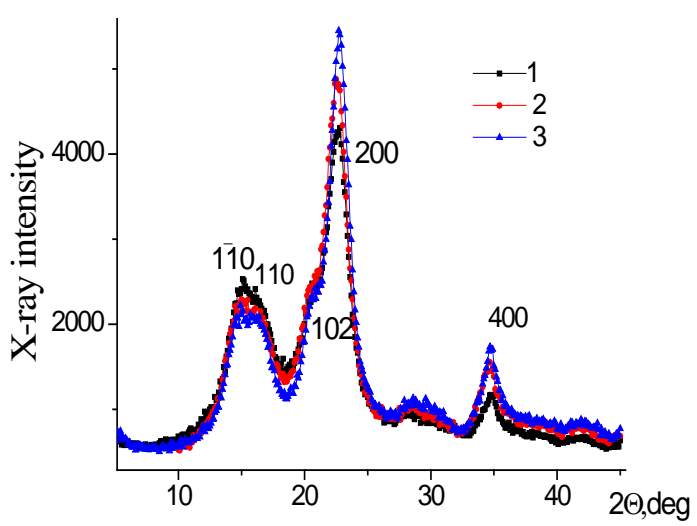

Figure 2: Diffractogram of samples from series A $(1: \xi=0.003,2: \xi=0.04,3: \xi=0.1)$ of scattered radiation intensity I on the angle $2 \theta$ between the axis of the counter and initial beam direction.

As can be noted in Figure 3, the peak positions do not change when the concentration of water increases. Thus, we can conclude that the water present in the lattice does not change the distance between the planes in the lattice.

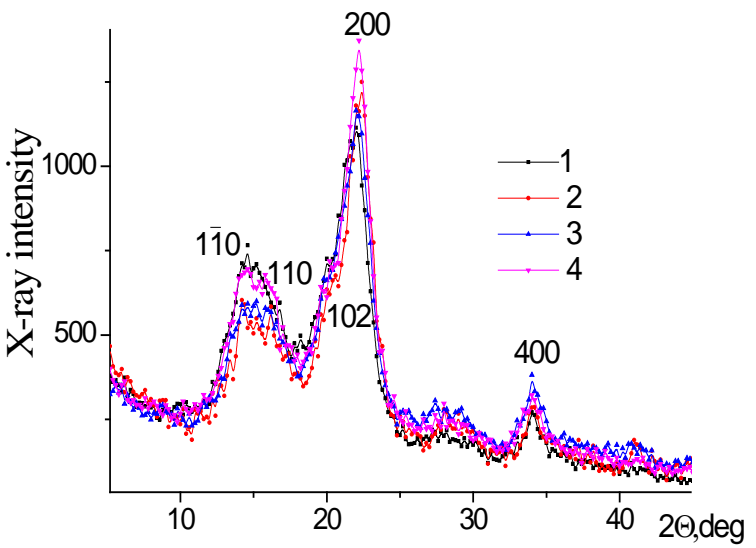

Figure 3: Diffractogram of samples from series B (1: $\xi=0.003,2: \xi=0.066,3: \xi=0.155,4: \xi=0.19$ )

Table 1

Structural characteristics of cellulose materials that contain water

\begin{tabular}{lccccc}
\hline Series & $\xi$ & $\omega$ & $b_{110}, \mathrm{~nm}$ & $b_{110}, \mathrm{~nm}$ & $\theta$ \\
\hline \multirow{3}{*}{$\mathrm{A}$} & 0.003 & 0.58 & 4.50 & 4.21 & \\
& 0.04 & 0.65 & 4.68 & 4.35 & 0.07 \\
& 0.1 & 0.68 & 4.91 & 4.62 & 0.18 \\
$\mathrm{~B}$ & 0.003 & 0.77 & 4.56 & 2.91 & \\
& 0.066 & 0.84 & 5.52 & 3.29 & 0.34 \\
& 0.155 & 0.84 & 5.52 & 2.98 & 0.23 \\
& 0.19 & 0.9 & 5.09 & 2.92 & 0.12 \\
\hline
\end{tabular}

In Table 1 , the peak width values $B_{110}$ and $B_{1 \overline{0} 0}$ are provided. The mentioned peaks correspond to the crystallographic planes (110) and (110). Our interpretation of these values is that an increase of the water amount in the samples leads to an increase of crystallite sizes. We think that this relation is the consequence of water molecules penetrating into crystallites. Obviously, water molecules will occupy the place between cellulose chains, in accordance with the principle that a system tends to the state with a minimum of energy potential. While the crystallite width changes, its size in the direction of the chain axis remains unchanged at the same time. This leads to the following formula for the relative volume change $\theta$ :

$\theta=\frac{b_{110}-b_{110}^{0}}{b_{110}^{0}}+\frac{b_{1 \overline{1} 0}-b_{1 \overline{1} 0}^{0}}{b_{110}^{0}}$

where values $b_{110}^{0}$ and $b_{110}^{0}$ characterize dried samples.

The calculated values of $\theta$ are shown in Table 1. 
When water is absent, $\omega$ is the mass degree of crystallinity. It is known ${ }^{9}$ that the value of this degree can be defined with the help of X-ray diffractograms. It is calculated as the ratio of the area under the peaks and the whole area under the diffractogram curve. We determined the values of $\omega$ for dried samples in this way, and they are shown in Table 1.
The same method was used for determining the $\omega$ value in the case when the samples contained a significant amount of water (significant compared to dried samples). At the same time, it is important to notice that, in the case of crystals with water, we cannot interpret $\omega$ as the mass degree of crystallinity.

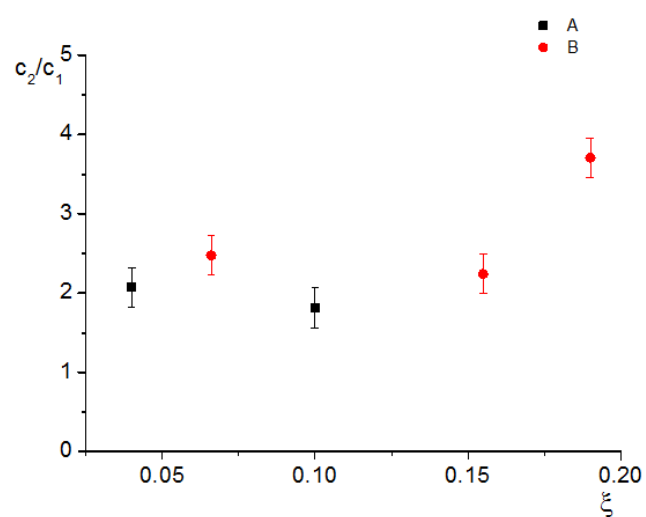

Figure 4: Water distribution between amorphous and crystalline phases of cellulose materials, when $\mathrm{T}=20^{\circ} \mathrm{C}$ (for systems $\mathrm{A}$ and $\mathrm{B}$ )

According to its definition, the mass degree of crystallinity is the ratio of the mass of the crystalline substance and the whole mass of a system. Water penetration into a crystallite leads to the emergence of defects in a crystallite's lattice and, as it was mentioned before, to an increase of the peak width on the diffractogram. However, a lattice does not disintegrate, in general, so a crystallite with water in it remains a crystallite, although it has defects. In this case, $\omega$, whose value we determined using the diffractograms, is the ratio of the mass of the substance in the defective crystalline areas (defective crystalline lattice plus the water in it) and the whole mass of the system (Eq. 5).

The values of $\omega$ for the samples containing water, which have been defined using the diffractograms, are shown in Table 1 . The values of the distribution index $c_{2} / c_{1}$, which was calculated using formula (15) and the data from the table, are shown in Figure 4.

Therefore, we draw the conclusion that, contrary to what is commonly believed, not only the amorphous region, but also the crystalline one can contain water, and the amount of water in the crystalline area can be significant. As can be seen in Figure 4, when $\xi \leq 0.15$, the water concentration in the crystalline areas $c_{1}$ is only twice smaller than the water concentration in the amorphous areas $c_{2}$. When $\xi>0.15$, the distribution index $c_{2} / c_{1}$ becomes higher. We suggest that this result evidences that when $\xi=0.15$, the water concentration in the crystalline areas reaches its limit; while when $\xi>0.15$, it remains constant.

\section{Structural model of water molecules distribution in crystalline areas of cellulose materials}

The values of $\psi_{i}$ in formula (3) are equal to:

$\psi_{i}=u_{i}-T s_{i}+p V_{i}, i=1,2$

where $\mathrm{p}$ is pressure, $\mathrm{u}_{\mathrm{i}}, \mathrm{s}_{\mathrm{i}}$ and $\Delta \mathrm{V}_{\mathrm{i}}$ are the energy, entropy and volume changes that are caused by the emergence of a water molecule in phase $i{ }^{8}$

We will consider that the value of $p$ is small enough to neglect the term $p V_{i}$ in expression 22 . This leads to formula:

$\psi_{2}-\psi_{1}=\left(u_{2}-u_{1}\right)-T\left(s_{2}-s_{1}\right)$

If we substitute the values of $c_{2} / c_{1}$ from the table in formula (4), we obtain:

$\psi_{2}-\psi_{1}=k_{B} T$ 
The model of an individual water molecule located in a crystallite lattice is shown in Figure 5

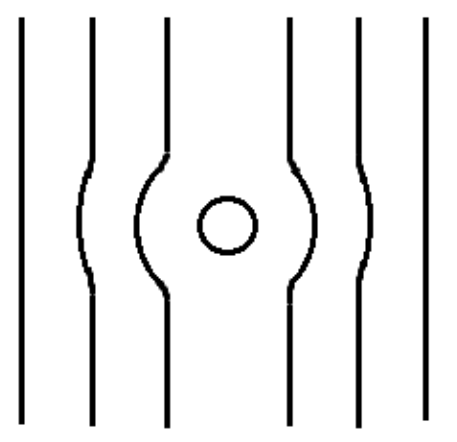

Figure 5: A water molecule in a crystallite lattice

Then, if we consider the water molecule as the point defect of a lattice, then $S_{1}$ is the entropy of the defect formation. Thus, its estimation was obtained: ${ }^{.3}$

$\left|S_{1}\right| \sim k_{B}$

Obviously, the entropy $\mathrm{S}_{2}$ has the same exponent, and it leads to the estimation:

$u_{1}-u_{2} \sim k_{B} T$

As mentioned above, the amorphous areas contain voids, and these mostly serve as locations for water molecules. The chains that surround a void are practically not deformed.

Figure 5 shows that when the water molecule penetrates into a lattice, the surrounding chains significantly deform. If we denote the energy of such deformation as $W$, the situation in Figure 5 is accordingly described by:

$u_{1}-u_{2} \sim W$

To estimate $W$, we will use the model "ball in a void", which is applied to point defects for this purpose. ${ }^{14}$ In this model, the penetrated molecule is a sphere with the radius $r_{1}$, which infiltrates into a spherical void with the radius $r_{0}<r_{1}$. Taking $\mathrm{r}_{1}-r_{0} \approx \mathrm{r}_{0}$, the following expression can be obtained for the deformation energy $W$ :

$W \approx 8 \pi G r_{0}^{3}$

where $G$ is the shear modulus of a medium that contains a void. (the water molecule is depicted as a circle, the chains are pictured as lines).

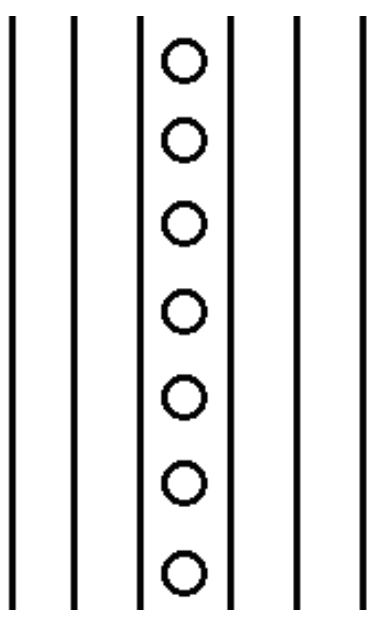

Figure 6: A cluster of water molecules in a lattice made by cellulose chains

Young's modulus of cellulose crystallites has the value near $10^{11} \mathrm{~Pa}^{15}$ If we accept this value, then we obtain the estimated value of the shear modulus $G \approx 10^{10} \mathrm{~Pa}$. Taking that $r_{0}^{3} \sim 10^{-24} \mathrm{~m}^{3}$, we get $W \sim 10^{-18} \mathrm{~J}$. This value is much larger than $k_{B} T \approx 4 \cdot 10^{-21} \mathrm{~J}$ at $\mathrm{T}=20^{\circ} \mathrm{C}$. In other words, the situation in Figure 5 contradicts expression 26, which was obtained using the experimental data.

One of the possible variants of water molecules location in a lattice, which would correspond to expression 26, due the minimization of chain deformation, is the formation of a cluster, which is a chain of water molecules. This chain is parallel to the cellulose chains (Fig. 6). Hydrogen bonds, which connect water molecules with each other and with the surrounding cellulose chains, contribute to the formation of such a structure.

\section{CONCLUSION}

The main conclusion of this work is that water molecules can penetrate the crystalline areas of cellulose materials in comparable amounts (with the same exponent of value) to the number of water molecules that infiltrate into the amorphous regions. The presence of water molecules in the crystalline regions of a cellulose material that contains water is imperative from a thermodynamics point of view. In addition to this, there are conditions in cellulose materials that provide a significant quantity of such molecules. 
One of those conditions is the possibility of formation of water molecule chains parallel to the cellulose chains. Importantly, the penetrated molecules, which break the hydrogen bonds between chains, then restore the hydrogen bond network, forming bonds with adjacent chains.

\section{REFERENCES}

1 A. Jamshaid, A. Hamid, N. Muhammad, A. Naseer, M. Ghauri et al., ChemBioEng Rev., 4, 1 (2017), https://doi.org/10.1002/cben.201700002

2 A. C. O'Sullivan, Cellulose, 4, 173 (1997), https://doi.org/10.1023/A:1018431705579

3 A. Ch. Khazraji and S. Robert, J. Nanomater., 2013, ID $\quad 409676$

(2013), https://doi.org/10.1155/2013/409676

4 J. C. Warwicker and A. C. Wright, J. Appl. Polym. Sci., 11, 659 (1967), https://doi.org/10.1002/app.1967.070110504

5 S. P. Papkov and E. Z. Fainberg, "Interaction of Cellulose and Cellulosic Materials with Water", Khimija, Moskow, 1976, 232 p.

6 Th. Angkuratipakorn, J. Singkhonrat and A. A. Christy, Key Eng. Mater., 735, 235 (2017), https://doi.org/10.4028/www.scientific.net/KEM.735.2 35

7 U. P. Agarwal, S. A. Ralph, C. Baez, R. S. Reiner and S. P. Verrill, Cellulose, 24, 1971 (2017), https://doi.org/10.1007/s10570-017-1259-0
8 L. D. Landau and E. M. Lifshitz, "Statistical Physics", $3^{\text {rd }}$ edition, Butterworth-Heinemann, 2013, 544 p., https://www.elsevier.com/books/statisticalphysics/landau/978-0-08-057046-4

B. Wunderlich, "Macromolecular Physics", Academic Press, 1973, 564 p., https://www.elsevier.com/books/macromolecularphysics-v1/wunderlich/978-0-12-765601-4

10 O. M. Alekseev, K. M. Kovalov, M. M. Lazarenko, M. V. Lazarenko, Y. E. Grabovskii et al., Cellulose Chem. Technol., 53, $15 \quad$ (2019), https://doi.org/10.35812/CelluloseChemTechnol.2019. 53.02

${ }_{11}$ K. M. Kovalov, O. M. Alekseev, M. M. Lazarenko, Y. F. Zabashta, Y. E. Grabovskii et al., Nanoscale Res. Lett., 12, 468 (2017), https://doi.org/10.1186/s11671017-2231-5

${ }_{12}$ M. Nedielko, S. Hamamda, O. Alekseev, V. Chornii, M. Dashevskii et al., Nanoscale Res. Lett., 12, 98 (2017), https://doi.org/10.1186/s11671-017-1862-x

13 A. C. Damask and G. I. Dienes, "Point Defects in Metals", Gordon and Breach Science Publishers, 1971

14 J. Frenkel, "Kinetic Theory of Liquids", Dover, reissue edition, 1955, $488 \mathrm{p}$.

15 A. Sturcova, G. R. Davies and S. I. Eichhorn, Biomacromolecules, $\quad$ 6, $1055 \quad$ (2005), https://doi.org/10.1021/bm049291k 